\title{
Fault Diagnosis-oriented Aeronautic Equipment IETM Knowledge Acquisition Method Study
}

\author{
Weiwei JIANG*, He YIN, Jin YAN, Xueqiao HOU, Liang ZHANG \\ Beijing Aeronautical Technology Research Center \\ Beijing, China \\ E-mail: jiangww0701@163.com \\ +* Corresponding author
}

\begin{abstract}
Interactive Electronic Technical Manual (IETM) serves as a key supporting technology of aeronautic equipment support informatization and is a carrier for structural, interactive and intelligent equipment technology data. In order to solve the problems that the traditional fault diagnosis system is single in diagnosis-oriented object, low in universality and insufficient in diagnosis knowledge and the like, accurate and efficient fault diagnosis can be performed by virtue of lots of fault diagnosis knowledge in aeronautic equipment IETM. However, knowledge source of the fault diagnosis knowledge exists in various systems of various stages of aeronautic equipment service life, knowledge acquisition model is established with respect to different fault diagnosis knowledge acquisition methods at various stages of study, and isomerism of each system is shielded, thereby realizing resource sharing, improving the fault diagnosis knowledge quality and providing more accurate fault diagnosis service for aeronautic equipment.
\end{abstract}

Keywords-IETM; fault diagnosis; knowledge acquisition

\section{INTRODUCTION}

With wide application in aeronautic equipment, various high technologies have seen their informatization degree continuously improved, so that it is increasingly difficult to perform fault diagnosis on the aeronautic equipment in the traditional mode, and multiple problems such as high cost, insufficient diagnosis knowledge, low efficiency and the like occur. Distant services, collaboration and intelligentization have become an important development direction and inexorable trend for study in the modern fault diagnosis field. Interactive Electronic Technical Manual (IETM) serves as a key supporting technology of aeronautic equipment support informatization and is a carrier for structural, interactive and intelligent equipment technology data[1]. IETM and remote collaborative fault diagnosis technology are combined, the universality and openness of the diagnosis system can be enhanced by exerting the advantages of IETM such as high information sharing, comprehensive and rich knowledge, high interactivity, etc., and the fault diagnosis accuracy and efficiency are improved. In order to rapidly and efficiently perform fault diagnosis, the key is to establish a knowledge base of the fault diagnosis system by taking equipment IETM resources in the grid as a main body, and quantity and quality of knowledge in the knowledge base determine the diagnosis capacity and diagnosis effect. Therefore, how to efficiently acquire systematic, authoritative and complete knowledge is a problem demanding prompt solution with respect to rapid and efficient fault diagnosis.

\section{AERONAUTIC EQUIPMENT IETM DOMAIN KNOWLEDGE STRUCTURE MODEL}

Domain knowledge is also known as background knowledge, comes from the field of artificial intelligence, and is mainly applied to some natural language understanding systems and knowledge-based expert systems. The domain knowledge refers to mutual relation among concepts in a certain field and sets[2] of constraints of related concepts.

Aeronautic equipment IETM fault diagnosis domain knowledge is a set of all the knowledge needed by fault diagnosis in IETM. The fault diagnosis domain knowledge is divided into explicit knowledge and implicit knowledge, as shown in Fig.1. 


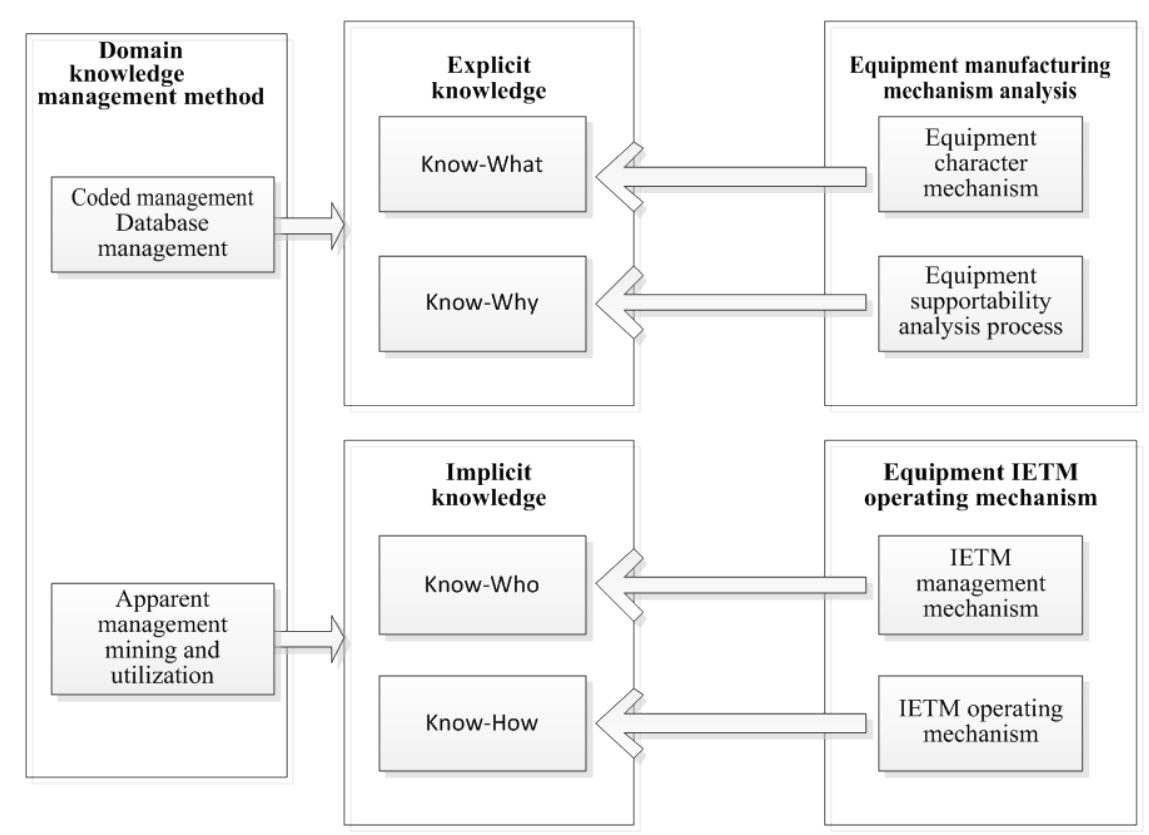

Figure 1. Aeronautic equipment IETM domain knowledge classification and relation

Explicit knowledge (Know-What) is contained in equipment manufacturing character mechanism, wherein Know-What is objective description of equipment itself and includes contents of the equipment, such as structural design, supporting characteristic design, function design, reliability, performance design, maintainability, process design, testability, material design, transportability, etc.; Know-Why is mechanism of integrated supportability engineering and integrated supportability, and can obtain equipment supportability data through related supportability analysis and supportability test and evaluation such as reliability and maintainability prediction, use and maintenance function design, fault tree analysis, fault mode influence and hazard analysis, battlefield damage mode influence and hazard analysis, maintenance analysis taking reliability as center, level of repair analysis, use and maintenance task analysis, etc.

Implicit knowledge is implicated in equipment IETM management and operating mechanism, wherein Know-Who refers to knowledge such as who to manage IETM, which department is primarily responsible, which department needs to cooperatively work, which aspects of experts need to participate in, etc. in equipment maintenance, support and personnel training; and Know-How refers to knowledge of using which resources in equipment IETM to complete equipment supportability task.

\section{Fault Diagnosis KNowledge ACQUisition REQUIREMENTS}

Knowledge acquisition is to refine explicit knowledge and implicit knowledge used for solving the problems from certain knowledge sources (including experts, books, database and people's experience).

Knowledge acquisition firstly needs knowledge source. Knowledge sources of equipment IETM are diversified.
With the further equipment supportability information construction, various equipment supportability and maintenance supportability information systems come into use in succession, considerable data is gradually accumulated in long-term system operating process, and fault diagnosis knowledge exists in knowledge sources of various service systems in data form. Lots of implicit knowledge exists in either fault diagnosis service engineering or service data and has not been discovered and utilized, or exists in brains of maintenance support personnel and experts in related fields and is difficultly acquired and utilized by other persons, so that sharing degree of equipment fault diagnosis knowledge is extremely low. Therefore, the implicit knowledge is rapidly, accurately and completely mined from various knowledge sources and saved in IETM in data module form, which is acquisition process of aeronautic equipment IETM fault diagnosis knowledge and precondition of improving fault diagnosis efficiency.

These fault diagnosis knowledge is different in forms and disperse in location, so its isomerism shall be shielded by utilizing related technologies, and the aeronautic equipment fault diagnosis domain knowledge is managed through IETM, thereby realizing continuous accumulation and sharing and reuse of the knowledge. The explicit knowledge is relatively easy to manage, while the implicit knowledge is difficult to manage. Management of equipment IETM explicit knowledge mainly refers to codification and database system to change the knowledge into information, while management of the implicit knowledge is mainly explicit and further mined and utilized, as shown in Fig.1. 


\section{Fault Diagnosis-ORIENTED IETM KNOWLEDGE ACQUISITION METHOD}

Knowledge sources which can be obtained at different stages of aeronautic equipment service are different, so fault diagnosis knowledge acquisition methods at various stages are also different.

\section{A. Fault Diagnosis Knowledge Acquisition Based on Equipment Design Knowledge}

At earlier stage of aeronautic equipment service, potential or direct fault diagnosis knowledge sources which can be obtained by IETM mainly include information of equipment design stage, equipment matched technology data, etc. In addition, if similar equipment fault diagnosis practice has been performed, there are some diagnosis experiences of similar equipment.

At this stage, although lots of information needed by equipment fault diagnosis is generated, the information is dispersedly distributed in various engineering models, and there is no equipment fault diagnosis service pathway, so relation between the information and equipment fault diagnosis must be analyzed, and useful information shall be extracted from the relation by refining, organizing and modeling, thereby realizing knowledge role conversion.

No matter how complicated the equipment is, weapon equipment is assembled by parts, so aggregation of knowledge of the parts forms knowledge body of the equipment. Therefore, method for acquiring fault diagnosis knowledge from equipment design knowledge is to abstract the parts of the equipment to knowledge units so as to describe diagnosis and maintenance information of the corresponding parts, and organization of different manners is performed on the basis according to different requirements. Aggregation of all the knowledge units is diagnosis knowledge model of the whole equipment. Model for acquiring fault diagnosis knowledge from equipment design knowledge is divided into three layers, that is, a physical layer, a logical layer and an application layer, as shown in Fig.2.

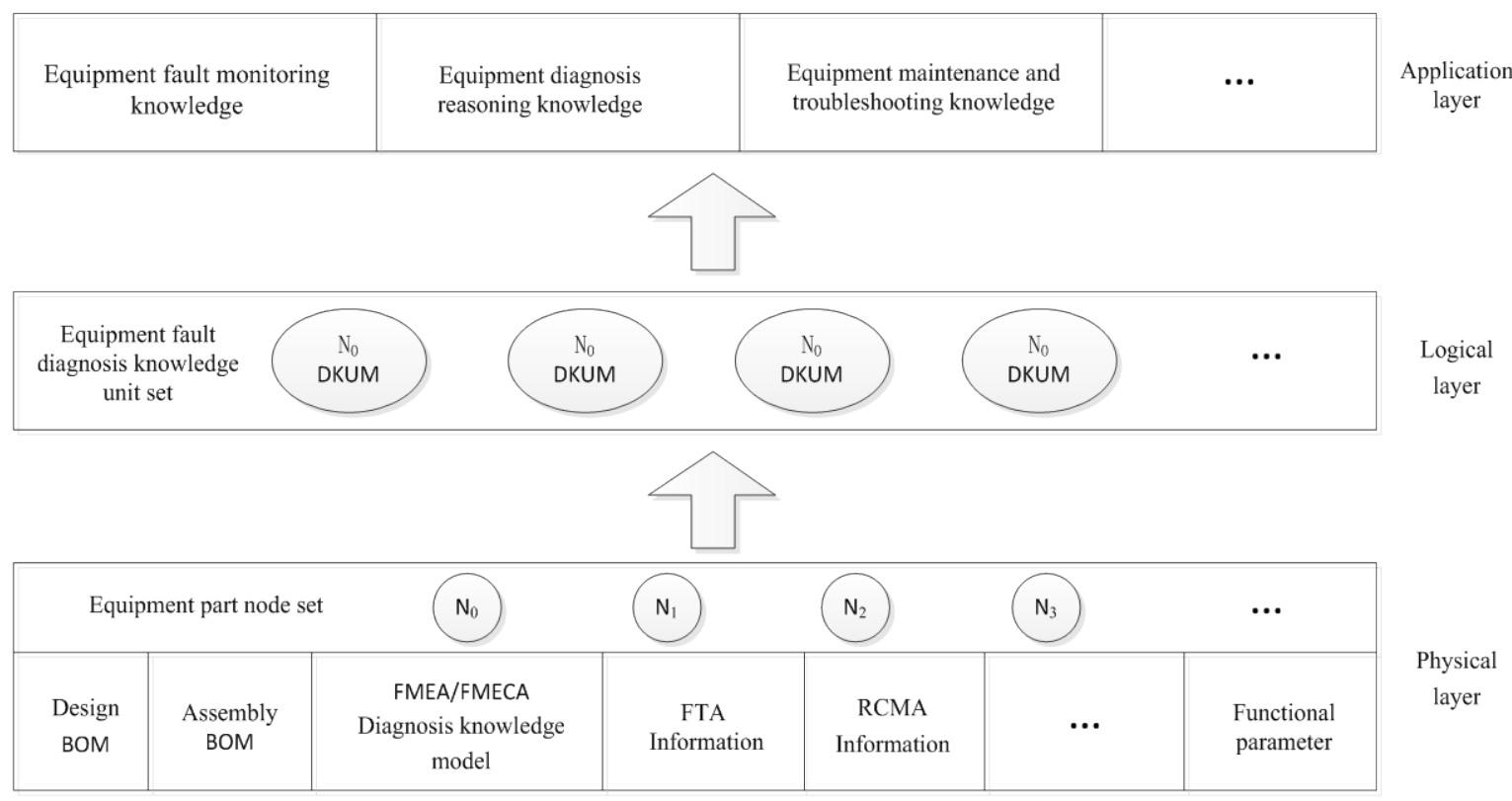

Figure 2. Fault diagnosis knowledge acquisition model based on equipment design knowledge

Information of various application fields in equipment design and manufacture process, such as equipment design BOM (Bill of Material) information, assembly BOM information, FMEA or FMECA information, FTA information, RCMA information, maintainability information, testability information, etc. is stored on the physical layer, and these information is physically stored according to multiple coded formats, generally including documents, graphs, database information, multimedia and the like.

Part node set of the equipment is abstracted to knowledge unit set on the logical layer, each part knowledge unit is defined as a diagnosis knowledge unit model (DKUM). DKUM refines, organizes and converts design knowledge needed by diagnosis of the parts into knowledge which can be used for equipment diagnosis from the whole life cycle of a single part, and information for design, diagnosis and the like is comprehensively and orderly stored in an integrated node information model in a centralized manner with respect to different requirements of various aspects of equipment fault diagnosis, so that the IETM or equipment diagnosis system can extract the needed information, thereby realizing conversion of design knowledge of the whole equipment to diagnosis knowledge.

The model can eliminate knowledge closeness at equipment design stage and use and maintenance stage, and finally, the knowledge can rapidly and effectively serve fault diagnosis at the equipment design stage. In the model, DKUM is a link connecting equipment design and fault diagnosis, is a key of acquiring diagnosis knowledge from 
design knowledge and controls flow direction and organization mode of these knowledge.

\section{B. Knowledge Acquisition Based on Heterogeneous Information System}

In the middle and later periods of equipment service, due to long-term learning and training, content, process and method of equipment fault diagnosis are known, and related diagnosis knowledge is gradually accumulated. With the further equipment supportability information construction, various equipment supportability management systems and equipment maintenance supportability service systems come into use one after another, thereby greatly enriching equipment fault diagnosis knowledge. To acquire knowledge from various expert systems, information management systems and integrated supportability information systems dispersed in the grid by taking IETM knowledge base as a main body is an important link. However, obvious heterogeneous characteristics may exist among the various information systems so as to cause heterogeneous information system data, so that IETM cannot smoothly acquire the knowledge from these systems. While the data serving as a carrier of knowledge base often contains valuable information to equipment fault diagnosis; by transforming the heterogeneous data, the knowledge can be transferred from various equipment supportability information systems into IETM so as to complete transformation of knowledge representation forms, thereby realizing knowledge acquisition based on heterogeneous information system. Therefore, knowledge acquisition based on heterogeneous information system is essentially to solve heterogeneous data transformation problem.
As an intermediate general metadata description language, XML has multiple advantages [3] of exchanging the heterogeneous data, and such advantages are just suitable for IETM to acquire knowledge from external information system. Basic principle and process of transformation of heterogeneous data resources are as shown in Fig. 3. It can be observed that non-IETM data resources can be simply divided into XML database data resources and relational database data resources according to the adopted database technology to which information systems belong. For the XML database data resources, due to query and transformation of XML data, IETMSchema specified in S1000D international norm serves as destination document structure specification and then is mapped into XML documents according with IETM technical standard. For the relational database data resources, XML data converter is utilized first, the IETMSchema specified in S1000D international norm serves as a destination document structure, the relational data is converted into XML according with provisions in the IETM technical standard. The whole conversion process is essentially the process of realizing data mapping, wherein non-IETM data resources serve as mapping source, and XML document according with structure requirements of IETMSchema serves as mapping target. After conversion completion, corresponding non-IETM data XML documents are stored into IETM knowledge base, so that knowledge acquisition based on heterogeneous information system is completed, the various knowledge converted from the heterogeneous information system can be conveniently managed, queried, called and extended, and the quantity and quality of the knowledge in the IETM knowledge base can be enriched and improved.

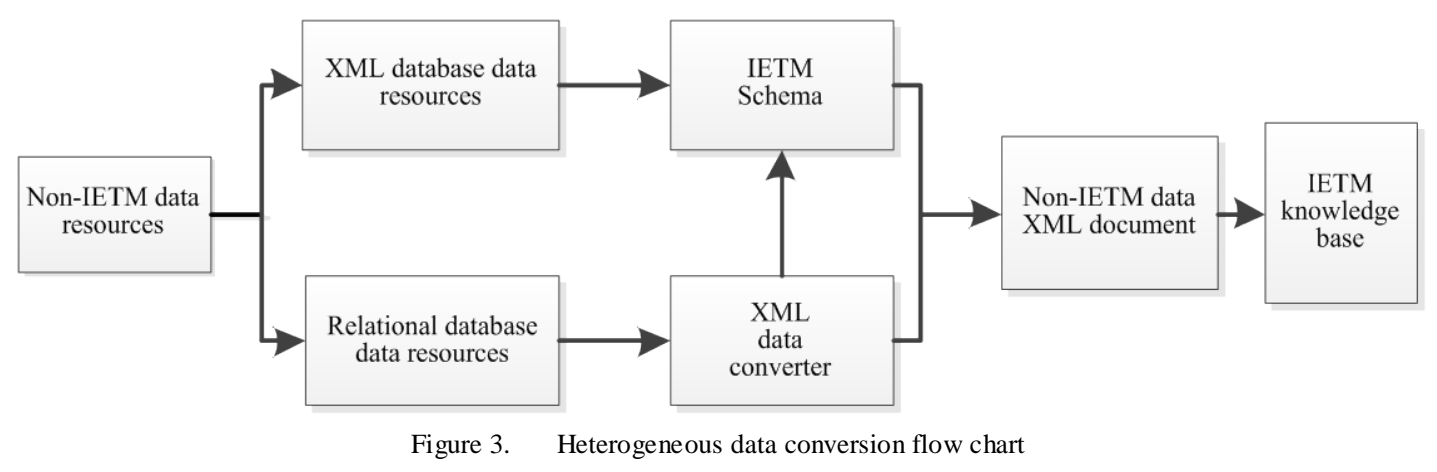

\section{Knowledge Acquisition Based on Information Feedback Mechanism}

In the later period of equipment service, due to specific working practice, equipment development manufacturer and army use and support personnel accumulate some successful diagnosis cases, summarize some diagnosis experiences and discover some new diagnosis methods. If these successful cases, experiences and methods can be timely and accurately fed back to IETM so as to implement dynamic update on IETM, systematic, comprehensive and authoritative IETM information knowledge can be guaranteed.
The purpose of the information feedback mechanism is to feed verification result back to diagnosis system after maintenance support personnel verifies diagnosis results given by the diagnosis system. With respect to fault problems which cannot be diagnosed, new solution is given by summarizing the previous experience and method, knowledge, cases and rules in the IETM knowledge base are modified, supplemented and perfected, and the accuracy of system diagnosis is continuously improved, so that experiences and methods in brains of equipment maintenance personnel and domain experts can be converted into equipment IETM knowledge. 
Working process of knowledge acquisition based on information feedback mechanism is that: equipment use and maintenance personnel store problems which cannot be solved by the diagnosis system into problem base through feedback module by virtue of interaction with IETM, then equipment manufacturer and fault diagnosis domain experts perform problem analysis and give diagnosis conclusions according to feedback information or field first-hand information obtained by communicating with use and maintenance personnel in real time. When the fault diagnosis conclusions are fed back to the system, system designer adds the fed-back diagnosis result into verification library. The use and maintenance personnel verify the diagnosis conclusions according to practice and experiment manners. If the fault diagnosis conclusions are verified to be wrong, the equipment manufacturer and fault diagnosis artificial experts will continuously handle the fault problem; and if the fault diagnosis conclusions are verified to be accurate, IETM creator and knowledge engineer convert the verified diagnosis conclusions into new fault diagnosis knowledge, cases or rules and update the IETM knowledge base together.

In the diagnosis process, army maintenance personnel, system designer, IETM creator, knowledge engineer, equipment manufacturer and domain experts all participate in the information feedback mechanism, their medium is the feedback module [4], users cooperate with one another by utilizing the feedback module, and self-correction and self-improvement of the system can be promoted.

\section{V.CONCLUSION}

Aeronautic equipment IETM is a new tool of fault diagnosis technology, and is required to have comprehensive, systematic and authoritative fault diagnosis knowledge. Lots of fault diagnosis knowledge is generated at various stages of life cycle of aeronautic equipment, and lots of knowledge is stored in each heterogeneous information system. Therefore, the method for acquiring fault diagnosis knowledge with respect to different stages of study has significances for creating high-quality IETM and improving fault diagnosis efficiency.

\section{REFERENCES}

[1] Zhang Fengming, Hui Xiaobin. Fault Diagnostics of Aeronautic Equipment [M] Beijing: National Defense Industry Press, 2010.

[2] Li Hua. Ontology-Based Emergency Domain Knowledge Representation and Reuse Research [D]. Doctoral Dissertation of Tianjin University, 2008.

[3] Zuo Weiming. Use-And-Query XML Data Markup Language Reference Manual [M]. Beijing: Posts and Telecom Press, 2007.

[4] $\mathrm{Xu}$ Zongchang. Equipment Supportability Engineering and Management [M]. Beijing: National Defence Industry Press, 2010. 